Ophthalmologe 2018 $\cdot 115: 195-201$ https://doi.org/10.1007/s00347-017-0602-z Online publiziert: 8. November 2017 (c) Der/die Autor(en) 2017, korrigierte Publikation November 2017. Dieser Artikel ist eine Open-Access-Publikation.

CrossMark

\author{
M. Thaller ${ }^{1,2} \cdot$ H. Böhm ${ }^{1,2} \cdot$ C. Lingenfelder ${ }^{3} \cdot$ F. Geiger ${ }^{1,2}$ \\ ${ }^{1}$ Abt. Zelluläre Biophysik, Max-Planck-Institut für medizinische Forschung, Heidelberg, Deutschland \\ ${ }^{2}$ Biophysikalische Chemie, Universität Heidelberg, Heidelberg, Deutschland \\ ${ }^{3}$ Pharmpur GmbH, Königsbrunn, Deutschland
}

\title{
Hyaluronsäuregele zur Druckregulierung in der Glaukomtherapie
}

entenabhängigen Faktoren, wie z. B. Alter, Hautfarbe und Voroperationen, auch von der Erfahrung des Operateurs und der Nachsorge mit Mitomycin C und 5-Fluoruracil abhängig. Neben der Trabekulektomie kommen auch die Lasertrabekuloplastik (SLT), die Kanaloplastik sowie minimalinvasive Chirurgie mit verschiedenen Stents zum Einsatz [2, 15, 17, 27]. Die SLT senkt den Augenausgangsdruck um maximal $30 \%$ und ist zeitlich begrenzt, kann allerdings wiederholt werden. Der Vorteil der Stents liegt im minimalen Eingriff, der zum Teil auch ambulant erfolgen kann, und in der geringer ausfallenden Nachsorge als bei der Trabekulektomie. Allerdings ist hier die Drucksenkung nicht so hoch wie bei der Trabekulektomie, und auch die Funktionsdauer ist bisher begrenzt. Zusätzlich kann es zu postoperativen Druckschwankungen und Bulbushypotonien kommen [27].

Mit dem Ziel, diese Problematiken anzugehen, erforschen wir daher einen neuartigen, auf Hyaluronsäure (HA) basierenden Stent zur dauerhaften Regulation des Augeninnendrucks. HA ist ein körpereigenes Polysaccharid, das aus den alternierend miteinander verknüpften Untereinheiten $\beta$-D-Glucuronsäure und N-Acetyl-D-Glucosamin aufgebaut ist [20]. Die unverzweigten HA-Moleküle kommen überall im Körper vor, hauptsächlich im interzellulären Raum z. B. als Teil der extrazellulären Matrix. Höhere Konzentrationen sind in Gelenkflüssigkeiten, im Gehirn sowie im Kammerwasser des Auges zu finden [5]. Auch im Trabekelwerk, das an der
Druckregulierung im Auge beteiligt ist, spielt HA eine essenzielle Rolle [16].

\section{) Hyaluronsäure ist ein körpereigenes Polysaccharid}

HA verfügt über die Fähigkeit, das 1000 Fache des Eigengewichts an Wasser zu binden. Damit können schon bei niedrigen Konzentrationen hochviskose und elastische Lösungen entstehen. Diese Eigenschaften sowie die gute Biokompatibilität haben dazu geführt, dass HA bereits in vielen Bereichen der Medizin wie der Orthopädie, derSchönheitschirurgie, der Wundheilung und auch der Ophthalmologie zum Einsatz kommt [1, 7, 9, 10, 26]. Chemisch modifizierte HA wird z. B. als viskoelastischer Körper- oder Weichgewebeersatz, als Trägermaterial für Arzneimittelfreisetzung, als Füllstoff für $\mathrm{Ge}$ webe, als Haut- und Gewebeschutz oder zur Gewebetrennung verwendet [14, 23]. Auch in der Glaukombehandlung kommt die HA bereits bei chirurgischen Eingriffen zum Einsatz $[11,28]$.

Neben unvernetzter HA haben auch die HA-basierten Hydrogele vermehrt Einzug in den medizinischen Bereich sowie in die Forschung gehalten [3, 24]. Hydrogele eignen sich durch ihre Biokompatibilität sowie antiinflammatorischen und nichtimmunogenen Eigenschaften hervorragend als Biomaterial in biomedizinischen Anwendungen oder im Tissue Engineering [4, 7, 12, 19]. Um ein HA-Gel zu erhalten, müssen die HA-Moleküle zunächst chemisch modifiziert werden (z. B. thioliert), sodass zesse zeitlich begrenzt und neben pati- 


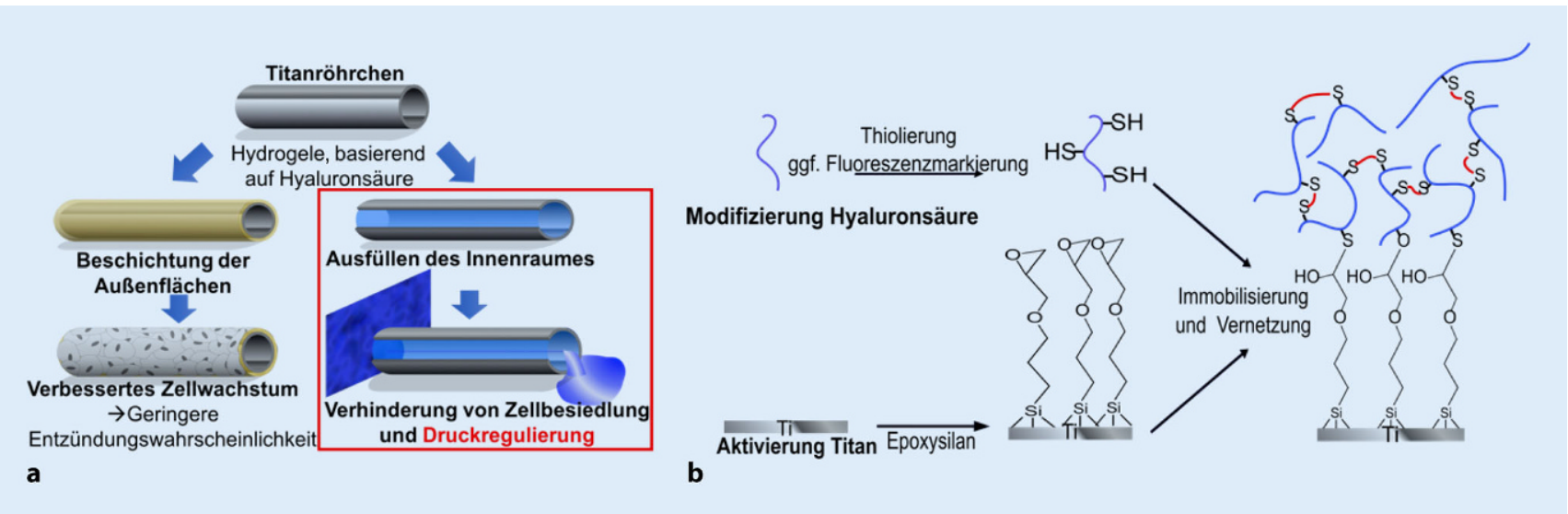

Abb. 1 a a Schematische Darstellung des neuartigen Stents und der Aufgaben des Hyaluronsäure-Hydrogels. b Schema zur Modifizierung der Hyaluronsäure und der Titanoberflächen zur Herstellung von immobilisierten Hyaluronsäuregelen an den Stentgrenzflächen. (Mit freundl. Genehmigung von Dr. Michael Thaller)

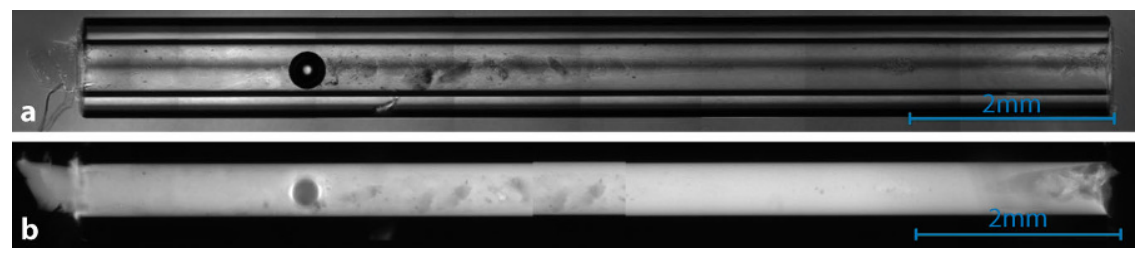

Abb. $2 \Delta$ a Phasenkontrast- und b Fluoreszenzaufnahme eines mit Hyaluronsäuregel gefüllten Glasmodellstents. (Mit freundl. Genehmigung von Dr. Michael Thaller)

sie anschließend durch einen Vernetzer miteinander verknüpft werden können $[3,29]$. Über die Art und Konzentration des Vernetzers sowie den Grad der Modifizierung und Konzentration der HA können die Geleigenschaften wie Festigkeit, Elastizität, Porengröße, Schwellrate, Perfusionsrate etc. eingestellt werden $[8$, $13,18,22]$.

In dem hier vorgestellten Implantat zur Behandlung des Glaukoms sollen HA-Gele mit unterschiedlichen Eigenschaften die bisherigen Probleme der Glaukombehandlungen lösen. Der Implantatkörper selbst besteht aus einem möglichst einfachen zylindrischen Hohlkörper aus Titan und soll zum einen mit einem HA-Gel beschichtet werden, das inflammatorische Prozesse verhindert und das Einwachsen durch gezielte Zelladhäsion verbessert. Zum anderen soll der Hohlkörper mit einem HA-Gel gefüllt werden, das die Druckregulierung übernimmt und zellabweisende Eigenschaften aufweist, um ein Zuwachsen oder Verstopfen des Implantats zu verhindern ( $\bullet$ Abb. 1a). Die hier vorgestellten Untersuchungen beschäftigten sich damit nachzuweisen, ob und wie das HA-Gel im Hohlraum zur Druckregulierung dienen kann und welche Parameter dabei eine Rolle spielen.

\section{Methoden und Ergebnisse}

\section{Modifikation von Implantaten mit Hyaluronsäuregelen}

Die Herstellung von Hydrogelen aus HA erfordert zunächst eine chemische Modifizierung, sodass die einzelnen HA-Moleküle durch Vernetzer verknüpft werden können und ein Hydrogel bilden. Hierzu wurde HA mit unterschiedlichen Molekulargewichten $\left(\mathrm{MW}_{\mathrm{HA}}\right)$ nach Shu et al. [29] thioliert, sodass zur anschließenden Gelbildung Vernetzer mit Acrylatgruppen verwendet werden können. In den folgenden Versuchen kam Hyaluronsäure mit einem Thiolierungsgrad (TG) von typischerweise $15-65 \%$ und N,N'Methylenbis(acrylamid) (MBA) als Vernetzer zum Einsatz. Die HA wurde zur besseren Beobachtung durch Fluoreszenzmikroskopie z.T. noch zusätzlich mittels N-Hydroxysuccinimid/1-Ethyl3-(3-dimethylaminopropyl)carbodiimid (NHS/EDC)-Chemie mit Fluorescein- amin markiert (Markierungsgrad FG $\approx$ $1 \%)$. Um einen Verbleib der Gele auf bzw. innerhalb des Implantats zu garantieren, müssen diese kovalent mit dem Implantatmaterial verknüpft werden. Hierzu wurden die Implantatoberflächen mithilfe von (3-Glycidyloxypropyl)-trimethoxysilane (GPS) mit Epoxygruppen modifiziert, an die sowohl natürlich vorkommende Hydroxy- als auch die zusätzlich eingeführten Thiolgruppen der HA binden können ( $\bullet$ Abb. 1b). Die Vernetzung der HA-Moleküle zum Hydrogel sowie die Immobilisierung wurden nach Optimierung der Einzelschritte zur Vereinfachung der Modifizierung der Implantate in einem Schritt durchgeführt (-Abb. 1b). Es konnten sowohl Titanimplantate (nicht gezeigt) als auch die für die Folgeversuche als Modellstents dienenden Glasröhrchen mit HAGelen bestückt werden ( $\bullet$ Abb. 2.) Die Hydrogele waren sowohl mit bloßem Auge sichtbar als auch ggf. im Fluoreszenzmikroskop. Gele, die in nicht silanisierten Röhrchen vernetzt wurden, entwichen aufgrund der fehlenden Immobilisierung schon bei leichtem Druck aus dem Röhrchen. Dies zeigt deutlich, dass eine kovalente Bindung der Gele an das Implantatmaterial essenziell ist, um als Ventil den Überdruck im Auge zu regulieren. 


\section{Untersuchung des Ventil- mechanismus}

Der Flüssigkeitsfluss durch die gelgefüllten Implantate wurde näher untersucht, um den Mechanismus aufzuklären. Hierzu wurden transparente Modellstents aus Glas, bei denen die gleiche Oberflächenchemie wie an Titanstents verwendet werden kann, herangezogen. Die $10 \mathrm{~mm}$ langen Glasröhrchen mit einem Durchmesser von $0,5 \mathrm{~mm}$ wurden, wie oben beschrieben, mit GPS silanisiert, und anschließend wurde das HA-Gel (TG = $\left.18 \%, 15 \mathrm{mg} / \mathrm{ml} \mathrm{HA}, \mathrm{MW}_{\mathrm{HA}}=60 \mathrm{kDa}\right) \mathrm{im}$ Inneren vernetzt und immobilisiert. Die Verteilung der Flüssigkeit beim Passieren des Implantats wurde im Fluoreszenzmikroskop beobachtet. Hierzu wurde eine Fluoresceinlösung mithilfe einer Spritze durch den mit einem HA-Gel gefüllten Stent gedrückt (- Abb. 3a). In der Mitte des Hydrogels bildete sich reproduzierbar und reversibel ein „Kanal“, durch den die Flüssigkeit floss (- Abb. 3b). Um das Verhalten des Gels genauer zu beobachten, wurde im nächsten Schritt ein fluoreszenzmarkiertes Gel (TG = $18 \%, 15 \mathrm{mg} / \mathrm{ml} \mathrm{HA}, \mathrm{MW}_{\mathrm{HA}}=60 \mathrm{kDa}$, $\mathrm{FG} \approx 1 \%$ ) verwendet. Unter einem manuell erzeugten Flüssigkeitsdruck einer physiologischen Salzlösung („balanced saline solution" [BSS]) konnte im Fluoreszenzmikroskop beobachtet werden, wie das Gel komprimiert wurde und sich in der Mitte ein Kanal bildete, der sich bei nachlassendem Druck wieder verschloss (- Abb. 3c-f). Dieser Vorgang war durch die elastischen Eigenschaften des HAHydrogels reversibel und konnte mehrfach wiederholt werden. Es konnte nicht für alle Proben eine Kanalbildung gezeigt werden. Bei einigen Proben kam es zu keinem Flüssigkeitsfluss, und bei sehr hohen Drücken entwich das Gel aus den Röhrchen. Um einen reproduzierbare Kanalbildung und damit einen Flüssigkeitsfluss zu gewährleisten, wurde die Präparation der Hydrogele dahingehend verändert, dass entlang der mittleren Längsachse des Gels die Vernetzung des Hydrogels aufgelöst wurde, sodass sich anschließend entlang dieser Linie in allen Röhrchen ein Kanal bildete. Die Vernetzung des Hydrogels wurde ent-

Ophthalmologe 2018 · 115:195-201 https://doi.org/10.1007/s00347-017-0602-z

(c) Der/die Autor(en) 2017. Dieser Artikel ist eine Open-Access-Publikation.

\section{Thaller · H. Böhm · C. Lingenfelder · F. Geiger}

\section{Hyaluronsäuregele zur Druckregulierung in der Glaukomtherapie}

\section{Zusammenfassung}

Hintergrund. Die steigende Zahl an Glaukompatienten und bei der Behandlung auftretende Probleme, wie z. B. Restenosen oder Hypotonie, erfordern neue Therapiemöglichkeiten, um das Erblinden der Patienten zu verhindern. Voraussetzung hierfür ist die Lösung der genannten Probleme mittels langlebiger Therapiemaßnahmen, um wiederholte Eingriffe zu vermeiden.

Fragestellung. Kann ein neuartiger Stent mit Hyaluronsäure-Hydrogelen (HA-Gel) als funktioneller Einheit zur langfristigen Druckregulierung und damit zur Therapie des Glaukoms eingesetzt werden?

Material und Methoden. Es wurden Modellstents, basierend auf HA-Gelen, hergestellt. Mit diesen wurde getestet, ob ein Druckausgleich möglich ist und welcher Mechanismus diesem zugrunde liegt.

Ergebnisse. Mithilfe der Modellstents konnte gezeigt werden, dass das HA-Gel im Inneren der Stents wie ein Ventil funktioniert. Unter einem bestimmten Gleichgewichtsdruck verschließt das HA-Gel den Stent und hält somit die Flüssigkeit zurück. Ab einem gewissen Überdruck ermöglich das HAGel den Abfluss der Flüssigkeit und führt zu einer selbstregulierten Einstellung des Gleichgewichtsdrucks.

Diskussion. Die hier vorgestellten Modellstents werden im nächsten Schritt miniaturisiert, sodass getestet werden kann, ob die Ventilfunktion auch in den für das Auge notwendigen Dimensionen funktioniert und damit das Problem der Hypotonie bei Glaukombehandlungen gelöst werden kann.

Schlüsselwörter

Hydrogele - Minimalinvasive Chirurgie . Druckausgleich · Stent · Ventil

\section{Hyaluronic acid gels for pressure regulation in glaucoma treatment}

Abstract

Background. The increasing numbers of glaucoma patients and complications occuring during treatment, such as restenosis and hypotony, require new treatment options to prevent blindness in patients. Therefore, the abovementioned problems should be solved to prolong the lifetime of implants and to prevent repeated surgery.

Objective. Can a novel stent with hyaluronic acid hydrogels (HA gel) as a functional unit be used to regulate pressure in glaucoma therapy in the long term?

Material and methods. Model stents were filled with HA gels and it was investigated if these could regulate the pressure and what the underlying mechanism is.

Results. The results of the investigations showed that the HA gel inside the stent functions as a pressure valve. Under certain equilibrium pressures the $\mathrm{HA}$ gel closes the stent and therefore retains the fluid. At a certain overpressure the $\mathrm{HA}$ gel enables the fluid to be released and leads to a selfregulated adjustment of the equilibrium pressure.

Discussion. The next step will involve miniaturization of the stents. Experiments will then show if the valve function will also work in the dimensions necessary for an eye implant and if the current problem of hypotension in glaucoma therapy can be solved.

Keywords

Hydrogels · Pressure regulation · Minimally invasive surgery $\cdot$ Stent $\cdot$ Valve weder mechanisch durch eine Glasfaser oder über einen Laser unterbrochen.

\section{Druckmessungen an Hyaluron- säuregel-modifizierten Implantaten}

Um Druckmessungen an den HA-Gel modifizierten Modellstents durchzuführen und damit den Einfluss unterschiedlicher Gel- und Implantatparameter zu 


\section{Leitthema}
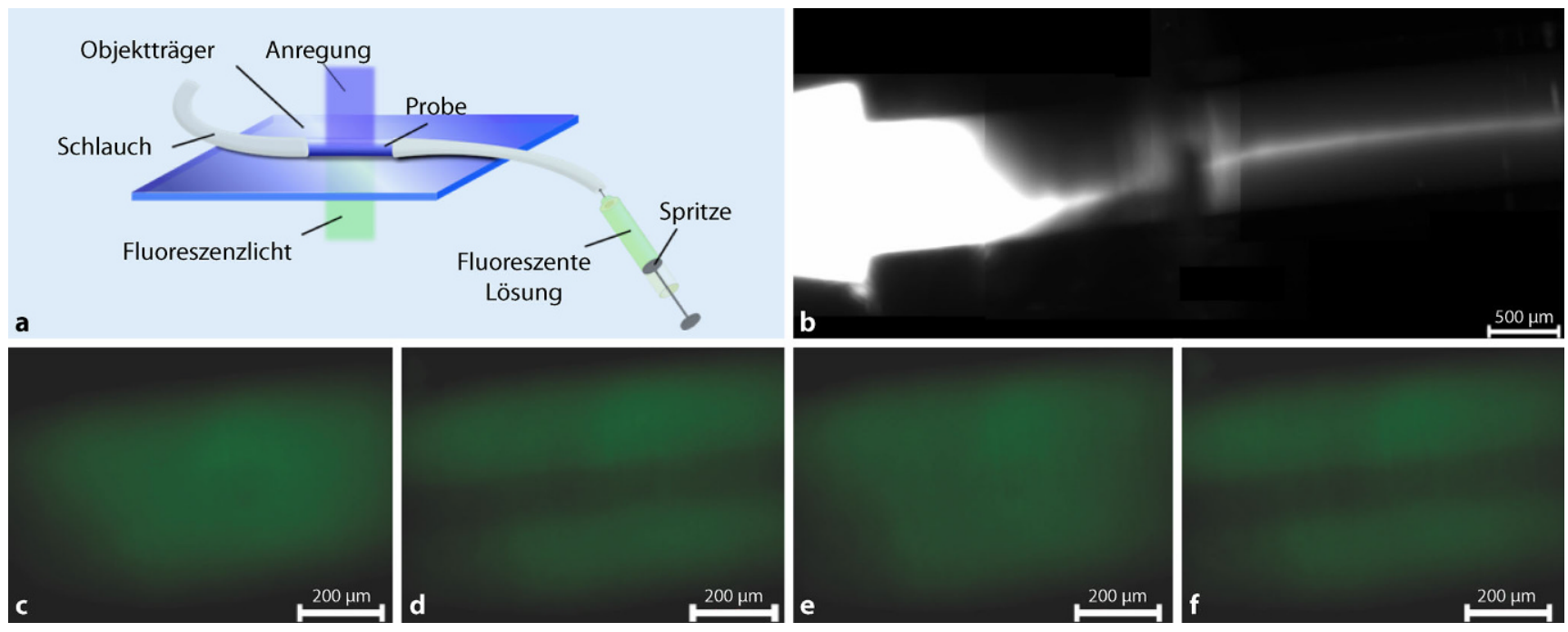

Abb. 3 A a Schematische Darstellung des Versuchsaufbaus zur Beobachtung des Gelverhaltens bei erhöhtem Flüssigkeitsdruck. b Fluoreszenzaufnahme eines Hyaluronsäuregels, nach dem der Druck erhöht wurde und fluoreszenzmarkierte Flüssigkeit durch das Gel hindurchfließt. c-f Zeitreihe von Fluoreszenzaufnahmen eines mit Fluorescein markierten Gels ohne Druck (c, e) und unter Druck (d, f). (Mit freundl. Genehmigung von Dr. Michael Thaller)

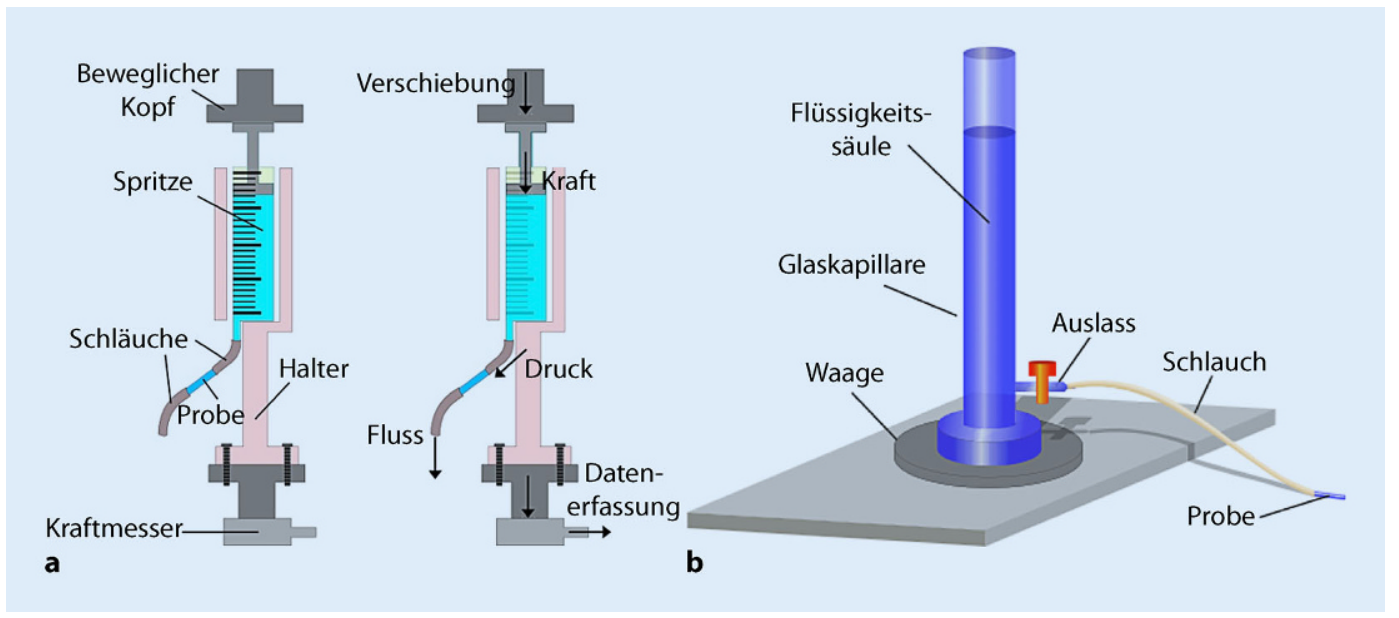

Abb. 4 \ Schematische Versuchsaufbauten zur Bestimmung von a Ausgleichsdrücken und b realen Gleichgewichtsdrücken. a Aufbau für dieZugprüfmaschine (BOSE), bei der durch kontinuierliche Verschiebung des Spritzenkolbens Druckerzeugt wird und eine Kraftmessung erfolgt. b Flüssigkeitssäule, bei der der hydrostatische Druck der Flüssigkeit auf die Probe einwirkt und über eine Waage bestimmt wird. (Mit freundl. Genehmigung von Dr. Michael Thaller)

untersuchen, wurden 2 Versuchsaufbauten konzipiert: eine schnelle Methode mithilfe der Zugprüfmaschine BOSE Electroforce III 3200 (BOSE ElectroForce Systems Group, Friedrichsdorf, Deutschland), mit der der Einfluss der HA-Konzentration und der Stentlänge an mehreren Proben gemessen werden konnte, und eine auf hydrostatischem Druck basierende Methode, die erlaubte, den realen Gleichgewichtsdruck für die Ventilfunktion der HA-Gele zu bestimmen (•Abb. 4).
Für die BOSE wurden die Proben an eine flüssigkeitsgefüllte Spritze angeschlossen, und anschließend wurde der Ausgleichsdruck ermittelt, bei dem sich ein konstanter Flüssigkeitsfluss bei Verschiebung des Spritzenkolbens ergab (• Abb. 5a, b). Mehrfachmessungen an einer Probe zeigten, dass der Ausgleichsdruck reproduzierbar ist, jedoch ein "Aktivierungsdruck“ notwendig ist, um das Hydrogel zu komprimieren, sodass sich der oben beschriebene Kanal für die Ventilfunktion bilden kann (- Abb. 5a, c). Der Ausgleichsdruck wur- de dann abhängig von Röhrchengeometrie und Geleigenschaften ermittelt. Zunächst wurde die Röhrchenlänge bei gleichbleibender Gelzusammensetzung untersucht. Hier zeigte sich, dass sich für längere Röhrchen ein höherer Ausgleichsdruck ergab (• Abb. 5d). Wurden höhere HA-Konzentrationen und damit kompaktere HA-Gele mit höherer Rückstellkraft, bedingt durch die räumliche Begrenzung, eingesetzt, stieg der Ausgleichsdruck ebenfalls mit steigender HA-Konzentration. 


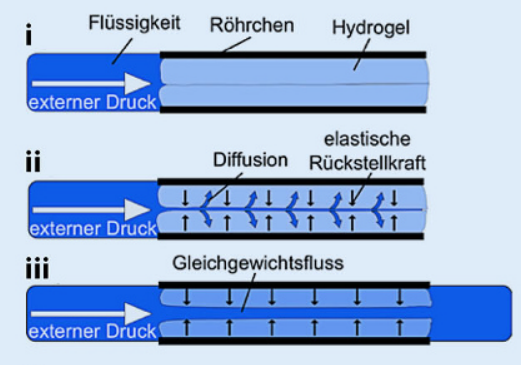

a
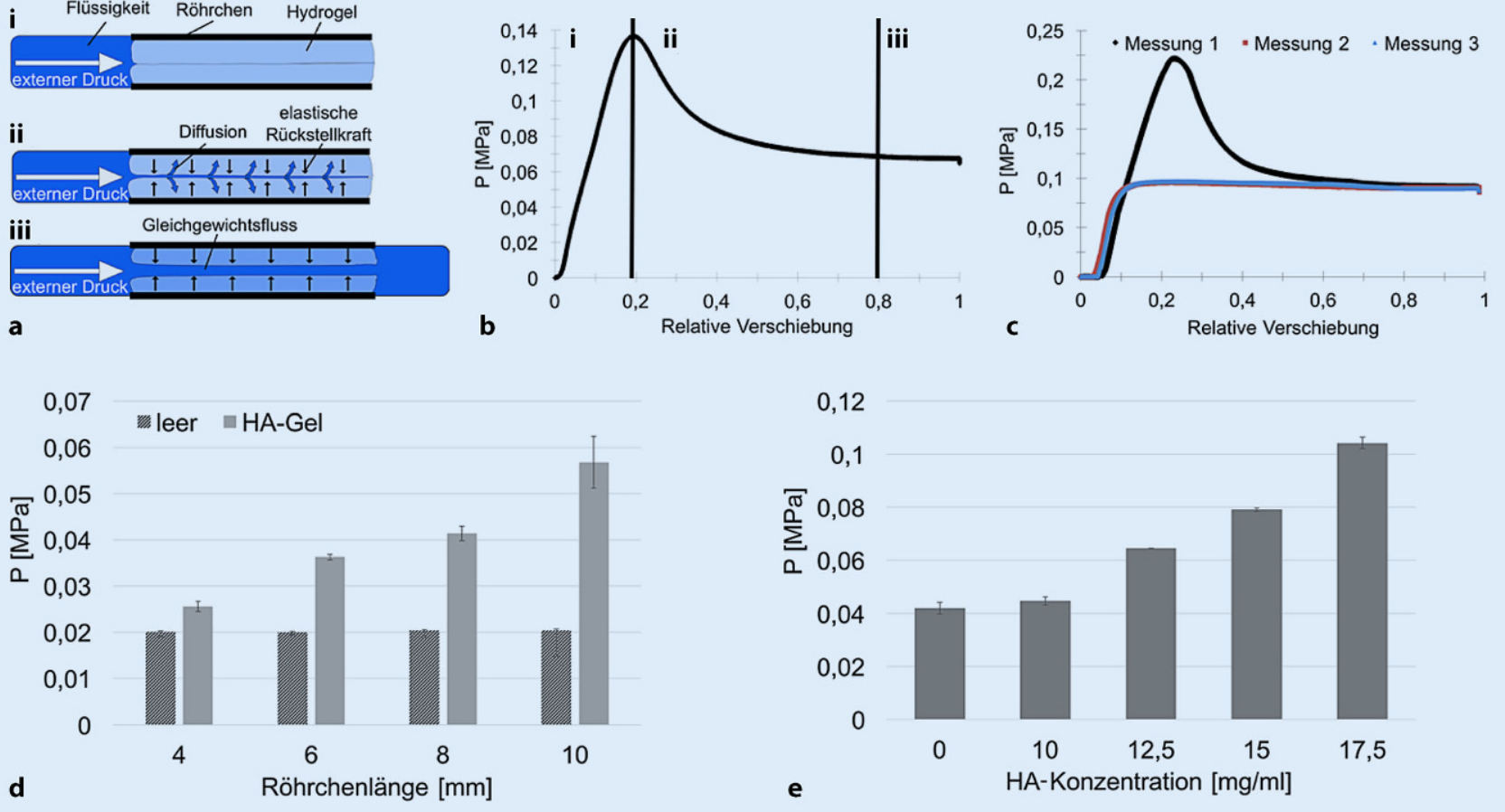

0,12

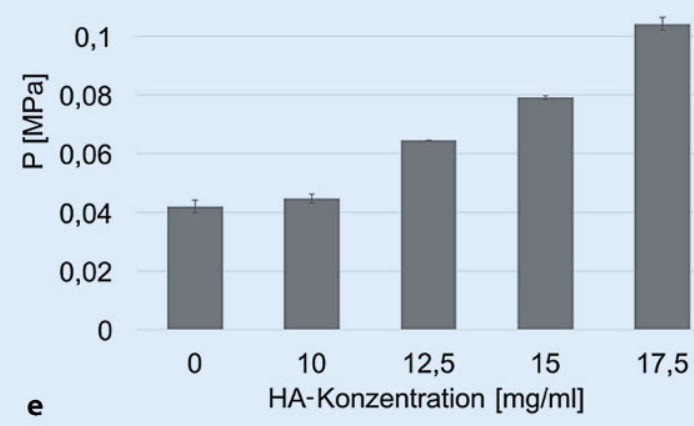

Abb. $5 \Delta$ a Schematische Darstellung des Gelverhaltens im Inneren des Stents, wenn ein externer Druck durch Flüssigkeit aufgebaut wird. b Typischer Kurvenverlauf einer "Druckmessung" an der BOSE mit Korrelation der unterschiedlichen Phasen zur schematischen Darstellung der Ventilfunktion in a. c Wiederholungsmessungen an einem mit Hyaluronsäure (HA)-Gel gefüllten Stent an der BOSE.d, e Ermittelte Ausgleichsdrücke in Abhängigkeit von d der Röhrchenlänge und e der HA-Konzentration. (Mit freundl. Genehmigung von Dr. Michael Thaller)

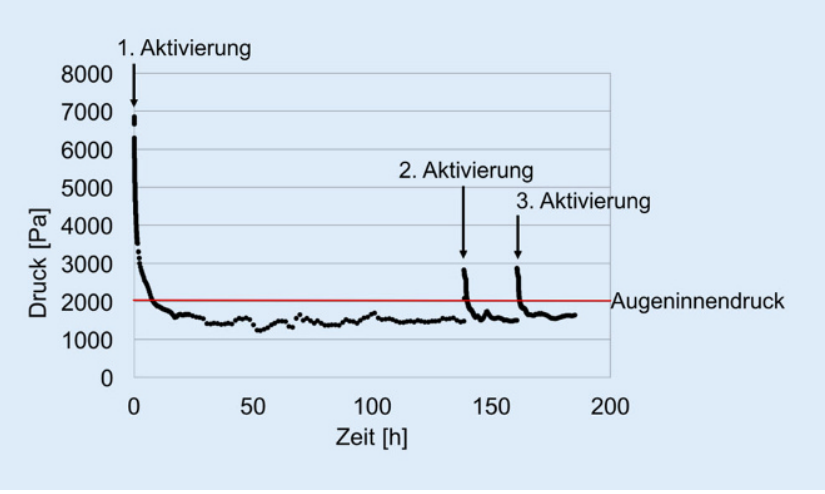

Abb. $6<$ Druckverlauf im Versuchsaufbau mit hydrostatischem Druck über mehrere Tage an einem mit Hyaluronsäuregel gefüllten Glasmodellstent. DerStent wurde insgesamt 3-mal aktiviert, wobei sich jedes Mal der gleiche Gleichgewichtsdruck einstellte
Der Druck, bei dem sich der Kanal wieder schließt bzw. der den realen Gleichgewichtsdruck darstellt, konnte mit dieser Methode nicht bestimmt werden. Dieser wurde anschließend in Langzeitmessungen mithilfe einer Flüssigkeitssäule über den hydrostatischen Druck ermittelt. Hierzu wurden aus technischen Gründen Glasmodellstents mit $1 \mathrm{~cm}$ Länge und einem Durchmesser von $0,5 \mathrm{~cm}$ benutzt. Diese wurden mit HA-Gelen $(\mathrm{TG}=52 \%, 15 \mathrm{mg} / \mathrm{ml}$ $\left.\mathrm{HA}, \mathrm{MW}_{\mathrm{HA}}=100 \mathrm{kDa}\right)$ gefüllt und vor dem Schwellen in BSS mit $100 \mu$ m oder $200 \mu \mathrm{m}$-Glasfasern entlang der Mittelachse des Glasröhrchens durchstoßen. Für diese Gele ergaben sich Gleichgewichtsdrücke nahe des erwünschten Augeninnendrucks von ca. $2000 \mathrm{~Pa}$ (• Abb. 6). Die Röhrchen konnten reproduzierbar mehrfache Überdrücke regulieren und waren dabei über mehrere Tage stabil.

\section{Diskussion und Ausblick}

Die bisherigen Forschungsergebnisse zeigen, dass eine Druckregulierung durch ein HA-Gel im Inneren eines zylindrischen Stents möglich ist. Der Gleichgewichtsdruck, bei dem keine weitere Flüssigkeit mehr durch den Stent fließt, ist dabei von der Geometrie des Stents selbst (Röhrchenlänge) sowie maßgeblich von der Zusammensetzung des HAGels, wie z. B. Kompressionsfähigkeit, abhängig. Das Hydrogel funktioniert hierbei wie eine Art Ventil. Bei Überdruck bildet sich im Inneren des Gels ein Kanal, durch den Flüssigkeit entweichen kann. Sinken der Druck und damit die Kraft auf das Gel, schließt sich der Kanal wieder, wenn die Rückstellkraft des Gels den Druck der Flüssigkeit übersteigt. Da die Rückstellkraft von der Gelkomposition abhängig ist, lässt sich der Gleichgewichtsdruck beeinflussen, bei dem das Ventil wieder schließt. 
Mit einem überdimensionierten Glasstent konnte gezeigt werden, dass der im Auge wünschenswerte Druck von ca. $2000 \mathrm{~Pa}$ durch Anpassung der Gelkomposition erreicht und ein Überdruck mehrmals hintereinander durch die Ventilfunktion des Gels ausgeglichen werden kann. Die Dimensionen der verwendeten Modellstents sind für einen Einsatz im Auge noch zu groß, sodass eine Miniaturisierung erfolgen muss. Hierzu müssen dann auch die Geleigenschaften neu angepasst werden. Da die HAGeleigenschaften jedoch durch mehrere Parameter wie HA-Konzentration, HAMoleküllänge, Vernetzerkonzentration etc. variiert werden können, wird es möglich sein, auch in kleineren Dimensionen den Augeninnendruck durch ein HA-Gel zu regulieren.

\section{》) Eine Druckregulierung durch ein Hyaluronsäuregel im Inneren eines zylindrischen Stents ist möglich}

Im nächsten Schritt müssen die zellabweisenden Eigenschaften der Gelkomposition, die eine Druckregulierung zulässt, untersucht werden. Das Gel im Inneren des Stents sollte ein Zellwachstum und Proteinablagerungen verhindern, sodass es zu keinem Zuwachsen oder Verstopfen des Ventils kommt. Hierbei könnten Vernetzer mit unterschiedlicher Ladung helfen, die dem Gel unterschiedliche Eigenschaften verleihen [8]. Um eine ökonomische Herstellung zu ermöglichen, ist angedacht, das bereits etablierte Verfahren des „Roll-up“ zur Herstellung von Titanröhrchen zu modifizieren [6]. Hierbei wird eine große Anzahl an Titanröhrchen durch ein Lithographieverfahren in einem Selbstassemblierungsprozess parallel hergestellt. Nach Integration einer HA-Schicht in das Verfahren wäre es möglich, mehrere hundert Stents parallel herzustellen. Andere Möglichkeiten zur Miniaturisierung des Stents bietet die Mikrofluidik, mit deren Hilfe kleine Flüssigkeitsmengen präzise gehandhabt werden können. Die Hauptaufgaben der weiteren Forschung an diesem neuartigen Stent liegen also in der Miniaturisie- rung, sodass ein Einsatz im Auge möglich ist, sowie in der Untersuchung des Zellverhaltens an den Grenzflächen des auf HA-Gel basierenden Stents.

\section{Korrespondenzadresse}

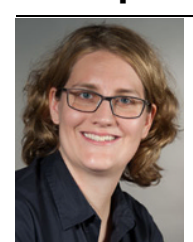

\section{Dr. F. Geiger}

Abt. Zelluläre Biophysik, Max-Planck-Institut für medizinische Forschung Jahnstr. 29, 69120 Heidelberg, Deutschland geiger@is.mpg.de

Danksagung. Wir danken dem Bundesministerium für Bildung und Forschung (BMBF; FKZ: 13N12372) und der Max-Planck-Gesellschaft für die Finanzierung des Projekts.

Funding. Open access funding provided by Max Planck Society.

\section{Einhaltung ethischer Richtlinien}

Interessenkonflikt. M. Thaller ist im Zusammenhang mit seiner derzeitigen Arbeit bei der Carl Zeiss Meditec Berlin mit der kommerziellen Entwicklung ophthalmologischer Implantate beschäftigt. H. Böhm, C. Lingenfelder und F. Geiger geben an, dass kein Interessenkonflikt besteht.

Dieser Beitrag beinhaltet keine von den Autoren durchgeführten Studien an Menschen oder Tieren.

Open Access Dieser Artikel wird unter der Creative Commons Namensnennung 4.0 International Lizenz (http://creativecommons.org/licenses/by/4.0/deed. de) veröffentlicht, welche die Nutzung, Vervielfältigung, Bearbeitung, Verbreitung und Wiedergabe in jeglichem Medium und Format erlaubt, sofern Sie den/die ursprünglichen Autor(en) und die Quelle ordnungsgemäß nennen, einen Linkzur Creative Commons Lizenz beifügen und angeben, ob Änderungen vorgenommen wurden.

\section{Literatur}

1. Altman R, Lim S, Steen RG et al (2015) Hyaluronic acid injections are associated with delay of total knee replacement surgery in patients with knee osteoarthritis: evidence from a large US health claims database. PLOS ONE 10(12):e145776. https://doi.org/10.1371/journal.pone.0145776

2. Brandao LM, Grieshaber MC (2013) Update on minimally invasive glaucoma surgery (MIGS) and new implants. J Ophthalmol. https://doi.org/10. 1155/2013/705915

3. Burdick JA, Prestwich GD (2011) Hyaluronic acid hydrogels for biomedical applications. Adv Mater 23:H41-H56

4. Day AJ, De La Motte CA (2005) Hyaluronan crosslinking: a protective mechanism in inflammation? Trends Immunol 26:637-643
5. Fraser JRE, Laurent TC, Laurent UBG (1997) Hyaluronan: Its nature, distribution, functions and turnover. JIntern Med 242:27-33

6. Giudicatti S, Marz SM, Soler L et al (2014) Photoactive rolled-up $\mathrm{TiO} 2$ microtubes: fabrication, characterization and applications. J Mater Chem C Mater OptElectron Devices 2:5892-5901

7. Goa KL, Benfield P (1994) Hyaluronic-acid a review of its pharmacology and use as a surgical aid in ophthalmology, and its therapeutic potential in joint disease and wound-healing. Drugs 47:536-566

8. Hagel V, Mateescu M, Southan A et al (2013) Desmosine-inspired cross-linkers for hyaluronan hydrogels. Sci Rep 3:2043

9. Hargittai I, Hargittai M (2008) Molecular structure of hyaluronan: an introduction. Struct Chem 19:697-717

10. Heden P, Sellman G, Von Wachenfeldt Metal(2009) Body shaping and volume restoration: the role of hyaluronic acid. Aesthetic Plast Surg 33:274-282

11. Higashide T, Sugiyama K (2008) Use of viscoelastic substance in ophthalmic surgery - focus on sodium hyaluronate. Clin Ophthalmol 2:21-30

12. Highley CB, Prestwich GD, Burdick JA (2016) Recent advances in hyaluronic acid hydrogels for biomedical applications. Curr Opin Biotechnol 40:35-40

13. Jeon O, Song SJ, Lee KJ et al (2007) Mechanical properties and degradation behaviors of hyaluronic acid hydrogels cross-linked at various cross-linking densities. Carbohydr Polym 70:251-257

14. Kogan G, Soltes L, Stern R et al (2007) Hyaluronic acid: a natural biopolymer with a broad range of biomedical and industrial applications. Biotechnol Lett 29:17-25

15. Kuerzinger GR, Eckert S (2010) Verfügbare Lasersysteme udn Wirkprinzip der Lasertrabekuloplastik. Ophthalmologe 107:8-12

16. Lerner LE, Polansky JR, Howes EL et al (1997) Hyaluronan in the human trabecular meshwork. Invest Ophthalmol Vis Sci 38:1222-1228

17. Manasses DT, Au L (2016) The new era of glaucoma micro-stent surgery. Ophthalmol Ther 5:135-146

18. Martini M, Hegger PS, Schadel N et al (2016) Charged triazole cross-linkers for hyaluronan-based hybrid hydrogels. Materials (Basel) 9(10):810. https://doi.org/10.3390/ma9100810

19. Naahidi S, Jafari M, Logan M et al (2017) Biocompatibility of hydrogel-based scaffolds for tissue engineering applications. Biotechnol Adv 35:530-544

20. Necas J, Bartosikova L, Brauner P et al (2008) Hyaluronic acid (hyaluronan): a review. Vet Med (Praha) 53:397-411

21. Oberacher-Velten I, Hoffmann E, Helbig H (2016) Volkskrankheit Glaukome. Ophtalmologe 113:746-751

22. Oelschlaeger C, Bossler F, Willenbacher N (2016) Synthesis, structural and micromechanical properties of 3D hyaluronic acid-based cryogel scaffolds. Biomacromolecules 17:580-589

23. Pfoerringer W, Pfoerringer D (2016) Arthrosebehandlung mit Hyaluronsäure und Kombinationen. Orthop Rheuma 19:30-32

24. Prestwich GD (2011) Hyaluronic acid-based clinical biomaterials derived for cell and molecule delivery in regenerative medicine. J Control Release 155:193-199

25. Quigley HA, Broman AT (2006) The number of people with glaucoma worldwide in 2010 and 2020. Br JOphthalmol 90:262-267 


\section{Correction}

26. Reinmüller J (2008) Hyaluronsäure in der ästhetischen Medizin.Historie, Entwicklung, heutige Bedeutung. JDtsch Dermatol Ges 6:S4-S9

27. Reznicek L, Boghos Y, Lanzl IM (2016) Möglichkeiten und Grenzen der operativen Glaukomtherapie. Ophtalmologe 113:833-837

28. Shaarawy T, Nguyen C, Schnyder C et al (2003) Five year results of viscocanalostomy. Br J Ophthalmol 87:441-445

29. Shu XZ, Liu YC, Luo Y et al (2002) Disulfide crosslinked hyaluronan hydrogels. Biomacromolecules 3:1304-1311

Ophthalmologe $2018 \cdot 115: 201$

https://doi.org/10.1007/s00347-017-0619-3

Online publiziert: 1. Dezember 2017

๑) Springer Medizin Verlag GmbH, ein Teil von Springer Nature 2017

CrossMark

M. Thaller ${ }^{1,2} \cdot$ H. Böhm ${ }^{1,2} \cdot$ C. Lingenfelder ${ }^{3} \cdot$ F. Geiger ${ }^{1,2}$

'Abt. Zelluläre Biophysik, Max-Planck-Institut für medizinische Forschung, Heidelberg, Deutschland

${ }^{2}$ Biophysikalische Chemie, Universität Heidelberg, Heidelberg, Deutschland

${ }^{3}$ Pharmpur GmbH, Königsbrunn, Deutschland

\section{Correction: Hyaluronsäuregele zur Druckregulierung in der Glaukomtherapie}

\author{
Correction: \\ Ophthalmologe 2017 \\ https://doi.org/10.1007/s00347-017- \\ 0602-Z
}

Sehr geehrter Leser,

sehr geehrte Leserin,

leider wurde der oben genannte Beitrag online zunächst mit fehlerhaftem deutschen Titel veröffentlicht. Wir bitten, den korrekten Titel Hyaluronsäuregele zur Druckregulierung in der Glaukomtherapie zu berücksichtigen und den Fehler $\mathrm{zu}$ entschuldigen.

Die Redaktion

\section{Korrespondenzadresse}

\section{Dr. F. Geiger}

Abt. Zelluläre Biophysik, Max-Planck-Institut für medizinische Forschung Jahnstraße 29, 69120 Heidelberg, Deutschland geiger@is.mpg.de
Die Onlineversion dieses Artikels wurde geändert:Der Beitragstitel enthielt einen Fehler.

Die Online-Version des Originalartikels ist unter https://doi.org/10.1007/s00347-017-0602-z zu finden. 